\title{
A Synergy of Ontology and Knowledge Classification for Decision Support Systems
}

\author{
Joe Essien \\ Faculty of Natural and Applied Science \\ Veritas University \\ Abuja, Nigeria
}

\begin{abstract}
Many problems within the software industry today emanate from the obliviousness that system development for decision support belongs to a category of problems that are classified complex or "wicked" in type. Therefore, procedural paradigms that address this phenomenon constitute areas of much exploration. Distinct with conventional problems, complex problems in information systems are problems that lack clarity in both their aims and solutions; lack definitive constructs and deficient of inherent logic that distinctively indicate when desired goals are achieved. This paper provides a discourse on the complex problems in Insurance Categorization. Ontology and knowledge classification in Insurance systems fall under this class of problems as insurance policies commonly describe problems exigent to unravel due to incomplete, contradictory and obstinately changing requirements. Because people exist in very unpredictable cultures with extensive categories of risk trajectories, it is commonly expected that individuals at some point in life are going to be challenged with situations which will hamper their privileges. Today, insurance businesses evolve either adversely or optimistically contingent upon the kinds of solutions they proffer for complex problems which may range from competition, management and pecuniary situation. This paper decomposes key functionalities of complex insurance processes to model knowledge-based ontology adopting standard Enterprise Architecture archetypes that impress upon the intricacies of insurance information systems management. The relationships between classifications, perspectives and knowledge are examined to promote the organization of homogenous entities intended for grouping procedures for decision support systems. The strengths and constraints of imperative categorization approaches are described in terms of their ability to ruminate, discover, and apply contemporary expositions through modelling so as to harmonize business goals with application infrastructures.
\end{abstract}

Keywords: Complex Problems, Decision Support Systems, Enterprise Architecture, Knowledge Management, Ontology.

\section{OVERVIEW OF COMPLEX PROBLEM}

In 1973, design theorists Horst Rittel and Melvin Webber propounded the term "wicked problem" to accentuate the complexities and challenges of addressing and planning dogmatic problems [1].Complex problems in information systems often described as "wicked problem", in consonant with Rittel [1], can be depicted as a communal or cultural concern that is problematic to effectuate and intrinsically difficult to unravel. Unlike simpler problems which can exult concise fixes and facile resolutions, these sorts of complex problems could also be interrelated and herculean to absolve. Complex problem refers to perceptions where there's no single definitive fix. Due to inherent complex interdependencies in these sorts of problems, an attempt to unravel one aspect may regressively reveal or generate other problems. Thus in creating the concept, design theorist Horst Rittel identified some common characteristics of complex problems [1]. These include lack of definitive formulation; lack of stopping rule for determining when a goal has been achieved; solutions to wicked problems are only good or bad (often used as description to software systems) but not true or false; and sophisticated as often used as portraiture to systems implementations. Each complex problem is exclusive to itself and its solutions are not explicit and cannot be accurately validated. This invariably accounts for the myriad software solutions that address homogenous business challenges everywhere in software solutions. Often those that attempt to solve complex problems are held liable for their actions. An endeavor whose solution requires an excessive number of individuals of varying mindsets and behavior to proffer a solution is probably going to be a wicked problem. Consequently, it is sensible to reflect on the roles of business analyst within organizations whose responsibility it is to understudy manual or semi-automated business processes so as to design and implement operational and efficient automated solutions. 


\section{REVIEW OF RELATED LITERATURE}

Many researchers who study insurance systems in comparative perspectives know that many national systems do not apply congruous models of automation. Many countries use a minimum of two, or more heterogeneous implementations. Thus it can be said that pure systems do not exist as many insurance systems use hybrid approaches. There has been broad consensus on this view especially among scholars [15], [16], [17], [18], [6]. Many countries adopt hybrid systems and matching all with one among the various ideal types is often difficult. Several authors have proposed solutions to this occurrence considering, for instance prevalent models [19], [20], [21]. Using the prevalent model as an expedient has a translucent practical value. But referring only to the prevalent pattern appears to be a distortion when certain countries with peculiar complex insurance systems are concerned [22]. In order to deal with the matter, it is essential to introduce into the conceptual scheme the aspect of categorization of insurance systems. By categorization, the presence of perceptions with reference to the overall system is decomposed into perspectives to which different models of healthcare organisation are applied. There are two basic categorization principles: i) categorization of insurance services; ii) categorization of the population. The categorization of healthcare services involves subdividing the whole range of insurance services into different perspectives [9]. An example of categorization of insurance services can ruminate extrications between essential procedures (i.e., those deemed necessary and thus to be included in the basic insurance package) and supplementary procedures (those not considered strictly necessary from insurance perspective [20]. There also can be categorization of exclusions. These are procedures associated with catastrophic risks or chronic and highly disabling conditions, the costs of which might be financially unsustainable for the majority of the population. The second sort of categorization concerns the population. This rationality of categorization involves the subdivision of citizens into divergent groups related to different insurance schemes. The population can be categorized according to different criteria. The most common are: occupation, earned income and age [17], [13]. Besides these, there are other possible criteria to segment the population; there may be programs predisposed exclusively to ethnic minorities and people affected by certain medical conditions, felons, expectant women, etc.

\section{KNOWLEDGE THEORY AND CLASSIFICATION}

Scholars in many fields, from philosophy to cybernetics, have long discussed the concept of data and therefore the problems of representing knowledge in information systems. Distinctions have been drawn between merely observing, perceiving, or maybe describing things and truly knowing them. To effective comprehend these distinctions implies a process of integration of facts about objects and therefore the context during which the objects and processes exist. Even in colloquial usage, knowledge about persons or artefacts are usually articulated in terms of deep relationships and meanings in place and space. Many researchers have delved into how theories are built, how knowledge is accumulated and shaped into a definitive representation [4], [5]. There are, of course, many processes involved, but often amongst them is the process of classification. Classification is the meaningful clustering of experience. The methods of classification are often utilized in a formative way and are thus useful during the preliminary stages of inquiry as a heuristic tool in discovery, analysis, and theorizing [6]. Once concepts are synchronized, the relationship among concepts becomes succinct. Classifications are often used as an upscale representation of what is known and is thus useful in communication and in generating a fresh sequence of assessment, contrast, and hypothesizing. Insurance information categorization is one among the oldest and most vital categorizations used for statistical purposes and as a grounding principle in decision support systems. Insurance often is an extremely competitive business that hinges on providing the proper products and industry-leading service to consumers. Obtaining and procrastinating on accurate data is critical to the success of the business. To overcome hassles with disjointed data across product lines, functions and channels, many insurance companies have designed information management practice to enable organizations to speedily gather and analyze data, whether it is pecuniary, claims, policy or consumer data. The principal motivation for classifying information is to condense the mass of raw data in such a way that similarities and dissimilarities are easily disassociated. Additionally, the system also points out the foremost significant features of the information at a glance.

Today, there are many insurance firms worldwide and thus there is an intensive challenge for insurers. Each organization looks for the simplest way of selling their insurance services within their ambiance targeting a specific group of people. During this work, a variety of existing modelling initiatives are applies to scale back service gaps and improve health outcomes for insurance groups. However, it is important to acknowledge that there are still more to be done. Thus, this work addresses an issue of whether the planning and modelling of insurance categorization 
system can help to market the role of consumers as partners in insurance delivery and advance the actualization of motivational goals for effective decision support. It has also been asserted that when consumers are involved in decision-making with reference to their insurance policies, it could result in better coverage outcomes and improved satisfaction. Thus practical coverage planning and style should evolve from a consumer perspective, with services grounded the needs of the consumer, instead of consumers being required to adjust to the desires of policy makers, planners and providers. Consumers should be supported and encouraged to participate in decision-making about their assessment and claims. This embraces enhancing knowledge, improving decision-making, particularly with reference to consumer preferences and social or other lifestyle factors which can increase consumer confidence in undertaking policies.

\section{SYNERGY OF CONTENT CLASSIFICATION}

The major aim of this work is to model and make reference models of Enterprise Architecture ontologies that might provide clarity to complex problems inherent in insurance categorization. The result of the work yields useroriented integrated insurance categorization archetypes that reduces ambiguity and supports systems development using any platform. This study is grounded on one case of ArchInsure (not real name) as an insurance firm that applies insurance schemes for various sectors to cover home, travel, automobiles and legal aid. The insurance information categorization models decompose, analyze and synthesize in aggregates insurance data to facilitate the management of insurance systems for effective decision support. More specifically, the ontologies prioritize action toward the building a consumer-focused integrated insurance categorization system that can increase goals realization, efficiency as well as improved quality, safety, performance and accountability of the insurance system.

There are many approaches to contextual classification and the development of motivation for classification schemes. Each category of classification process has diverse goals, similar and dissimilar structural properties, and different strengths and weaknesses in terms of data representation and knowledge discovery. A representative approach of classification by hierarchies and structures is discussed with an inherited understanding of hierarchical classifications from Aristotle [7]. Aristotle posits that every nature of categorization is comprised a unified whole. The entire concept can also be subdivided, into "natural" classes, and every class further into subclasses. This process follows an orderly and systematic set of rules of association and distinction. During a pure hierarchical classification, membership in an allocated category of class is decided by rules of inclusion referred to as necessary and sufficient criteria. To belong to a category, an entity must have the prescribed (necessary) attributes; if it is the required attributes, this then constitutes sufficient variants to assign the entity to the category. Due to these formal properties, hierarchical classification schemes are yet to be relevant in knowledge representation and discovery for several reasons. A hierarchical classification is typically a reasonably comprehensive classification since all rules for synergy and distinction must be made in advance. This suggests that, before the structure is established, the system designer must have good knowledge about the extent of the entities, their qualities, and therefore the key criteria along which they are comparable and dissimilar. The formalism of a hierarchy allows a simple representation of the heterogeneous complex attributes. Much information is often carried by the hierarchical data structure. For this reason, a hierarchy allows reasoning from incomplete evidence. This is often especially useful if the shared criteria are not apparent or easily observable. Hierarchical classification enables realistic definitions, which are deemed to be superior to other sorts of definitions as they define ways of expressing how an entity is like another, and also how it differs in some way. The strength of this delineation lies in its ability to succinctly describe a peculiarity of attributes of affinity and a crucial aspect of distinction. If the standards by which the classificatory structure is made are theoretical within the sense that they unravel fundamental and meaningful distinctions, then the classification scheme as in its entirety provides a visualization of the phenomena it is representing. Such a perspective enables recognition of overall frameworks and anomalies, pragmatic or problematic relationships. A holistic high-level view is typically a trigger for knowledge generation, allowing the researcher to visualize the individual instances to ascertain their congruity into a bigger context. In theory, the central role of the adverse selection problem within the insurance market is unclear, as this is essential to correct market failure and anomalies in classification method. From an empirical point of view, with reference to the role of classification as a remedy for asymmetric information consequences, Wendt et al. [18] found evidence that the prohibition of risk classification in insurance markets forces safer policies. 


\section{RATIONALE FOR CATEGORIZATION}

Categorization has an extended standing tradition in science since processes of sorting, ordering, and comparing involved in classifying social, political, or economic entities are intrinsically scientific: "By making such categorization, generalizations regarding the members or properties of given categories also are made possible. Consequently, categorization is often regarded as the foundation of all science [12]. Further, it has been asserted that categorization can contribute to the enactment of hypotheses about the consequences of welfare states in terms of political cleavages or market performance [14], [15], [19]. This provides a conceptual frame for description and a reference scheme for comparisons across societies. Furthermore, categorization can yield new perspectives and theoretical approaches that foster validation of other hypotheses. The generation of types is often inductive and can also support many features of real cases derived deductively from theoretical concepts. The inductive strategy depends strongly on the sampling of cases. It therefore bears the risks of designing too narrow classes which fail to involve all theoretically possible types and of reducing generalizability. Conversely, deductive approaches are often too abstract and can dishevel the link to the critical features distinguishing actual cases [7]. Either way, categorizations require the definition of criteria to differentiate cases. The event of those criteria and therefore the following steps of arranging the cases will contribute to the understanding of new cases, their differences and similarities. A longtime method to construct classes is described by Iversen et al [15]. Iversen et al. states that ideal types are developed on the idea of real cases by revealing their typical features and arranging these and other more diffuse aspects into a unified analytical construct [15]. The comparison between these ideal types and real-historical cases could lead to a method for case instances, the differences between them, and changes over time. As such, the ideal-typical method may be a central start line for the measurement of change [18]. Categorizations often involve the peril of concept stretching and forcing diverse cases into an equivalent class, which can lead to producing of a meaningless typology. However, these disputes often foster further research: the event of typologies and therefore the ambivalent categorization of cases have acted as a catalyst for comparative research, as are often seen from the spread of state literature and therefore the effects of early health systems taxonomies [19]. Besides provoking research questions associated with categorization, like why some cases deviate from the perfect type, there is further use of categorizations in guiding case selection. Thus, Wendt et al. state: "The possibility in pinpointing those cases which are of greatest relevance can help guide the case selection of researchers that do not conduct large studies" [18]. Therefore, comparative research methods that adopt similar/different systems approach often make use of categorizations since it can control be effective in control of context variables. While divergent developments of cases belonging to an equivalent type may cast doubt on the categorization, this finding also can help to spot the variables which drive the divergence.

\section{ARCHITECTURAL DESIGN PATTERNS}

In software engineering, a design pattern may be a general reusable solution to a commonly occurring problem in software design. A design pattern is not a finished design to be transformed directly into code. It is a framework that defines a way to solve a problem. Object-oriented design patterns typically show relationships and interactions between classes or objects, without specifying the ultimate application classes or objects that are involved. Design patterns reside within the domain of modules and interconnections. At a better level there are architectural patterns that are larger in scope, usually describing an overall pattern followed by a whole system. There are numerous types of design patterns; Structural patterns address concerns associated with the high level structure of an application being developed; Computational patterns address concerns associated with the identification of key computations; Algorithm strategy patterns address concerns associated with high level strategies that describe the way to exploit application characteristic on a computation platform; Implementation strategy patterns address concerns related to the realization of the source code to support how the program itself is organized and the common data structures specific to parallel programming and; Execution patterns address concerns associated with support of the execution of an application, including the strategies in executing streams of tasks and building blocks to support the synchronization between tasks. Design patterns can speed up the development process by providing tested, proven development paradigms. Effective software design requires considering concerns that may not become evident until later in the implementation. Reusing design patterns helps to avert subtle issues that can cause serious problems, and it also improves code readability for coders and architects who are conversant with the patterns. In addition to this, patterns allow developers to communicate using well-known, implicit names for software interactions. In order to achieve flexibility, design patterns usually introduce additional levels of indirection, which in some cases may obscure the resulting designs and impede the application performance. By definition, a pattern must be programmed into each application that uses it. 


\section{HANDLING COMPLEX PROBLEMS MODELLING}

The design and integration of multifaceted software-defined services that use the online services can be construed as a growth from previous models of software models, and therefore becomes a complex problem. DeGrace and Stahl (1990) illustrated on the concept of complex "wicked" problems to software modelling. In the last era, other experts have pointed out that software modelling shares many attributes with other design paradigms. Principally, people, process, and technology problems have combined Rittel's [1] notions into their software modelling methodologies [9], [10]. Many problems in the software industry today result from our unawareness that system development belongs to a class of problems that are inherently complex. As a result, new process paradigms are needed. Rittel opines that its methodical, collaborative approach could provide methodical, solution-oriented problem-solving. It syndicates people with diverse backgrounds, skills, and experience that work collaboratively in a process-based approach to unravel intricate issues [1]. A mixture of systems thinking and responsive methodologies can help to tackle complex problems. System designers have proceeded to highlight how to exploit systems thinking when confronted with complex modelling problems. System thinking is the process of understanding how constituents of a system stimulate each other as well as other systems, therefore perfect for complex problems solving. System thinking is enhanced when pooled with agile methodology, an iterative approach to design and product development. Agile methodology supports solutions modelling and in a concerted environment, breeds effectiveness and actualization of the user's changing requirements. Collectively, systems thinking and agile methodology leads to a better solution modelling as they both evolve with complex problems. Thus an amalgamation of systems thinking and agile methodology inspires the exploitation of practices and sharing with others so that future iteration of the design process can be accomplished. Instead of pursuing a solution that totally eliminates a complex problem, it has been recommended that one should distinguish those actions that occur in an on-going process, and advance actions needed to ameliorate outcomes [14].

\section{THE ARCHINSURE COMPLEX PROBLEM}

The research data used in this study represents complex problems case obtained from a single systems design initiative of ArchInsure Insurance Company. The singular study was carried out in a specific context of home, travel, auto and legal aid with focus on standard documentation found in that sector. The existing system used and identified is paper-based methods/processes that have resulted in a lack of effective connectivity among the major stakeholders in the scheme. This led to a number of problems such as delay of registration and update, errors in the manipulation of consumer data and claims payment, duplication and misrepresentation of data, delay in the delivery of reports and long waiting period in consummating of claims Many flowcharts and workflow diagrams were obtained which demonstrated the step by step approach for the registration process for individuals who utilize the insurance service application. This phase helped to solicit knowledge that led to the identification of the complex problems in the current system and how to convert the requirements into conceptual solutions that could set a baseline for design of the models. It also facilitated the identification of the design needs, examined the pertinent techniques and proposed a design solution. One of the main functions of ArchInsure is selling of insurance services. The insurance firm maintains manual records of policy holders, claims and policy purchase documentation leading to a variety of convoluted problems. These include difficulty in locating files, poor service delivery due to time wasted in processing claims and other vital documents, delay in obtaining information, inefficient workflow procedures and many other sharp practices. As a result of the few downsides faced with the existing system, there would be a need to design a system which would be able to address them. Information will be styled in the proposed system to fit customers' needs and achieve business goals. The online system will create a means of delivery channel by supporting all phases of web-based application from contacting to contracting. It will serve as universal medium that enables insurers to enter new markets in a simple and efficient way. The online system should be capable of reducing cost in many ways including expediting administrative procedure. The functionalities of the web-based solution will include the capacity to start new policy purchase, access to information about their policy, ability to register existing customers' contact details on the site, and an informative system that includes relevant policy information about insurance services provided, its departments, its history and mission. User should be able to view their own personal details when login into the Policy Holder module.

This insurance management system should have facilities like search tools for insurance awareness articles, guidelines, illustrations through images for visitors. This insurance management system should competently manage the company, records; deliver instant access and one that improves the productivity. In this online process the user should be able to access the system and obtain details about insurance and its categories. Also it should show the details about different interval schemes to corresponding insurance type or insurance policy. The main objective of the proposed system is to allow extensive 
categorization to solve as much as possible the complex problems identified. The system should also be flexible to allow interoperability with other internal and external systems.

\section{THE ARCHINSURE MODELING}

The ArchInsure encompasses a set of viewpoints, notations and concepts that can be used to construct high quality insurance application. It is a set of logical and integrated ontologies for generating, evaluating and specifying concepts for an insurance system with structures and functions aimed to achieve client's objectives or users' needs while satisfying a specified set of goals and constraints.

\subsection{The goal realization perspective}

The goal realization perspective models the motivation of high level goals and drivers into more specific outcomes, and the refinement of these distinct outcomes further into requirements or constraints. Figure 1 shows the factoring of goals into subgoals using the aggregation, while the refinement of goals into requirements is modeled using the realization.

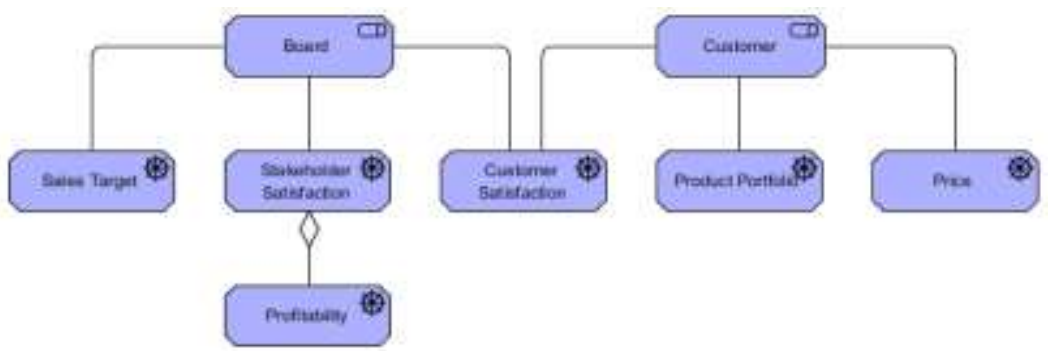

Figure 1: ArchInsure Goals Realization Perspective

The goal realization perspective allows modeling of refinement of high-level goals into more tangible goals, and the refinement of tangible goals into requirements or constraints that describe the properties needed to realize the goals.

\subsection{ArchInsure Actors Cooperation Perspective}

The ontology adopts a top-down approach which deploys a stepwise design to fundamentally disintegrate the system to gain insight into its compositional sub-systems. The approach takes into account all related artefacts of the system that needs to be modeled from different stakeholder and organizational perspectives with respect to four key concerns namely; i) capability to manage problem complexity through decomposition of constraints; ii) capability to produce abstract exemplification of design choices through categorization; iii) capability to proffer explicit meaning to concepts and notations used in the design; iv) capability to establish guided paths to realize precise end-user tasks so as to reduce complex problems and v) adoption of a standard modelling tool and strategy most appropriate for rationalizing complex problems.

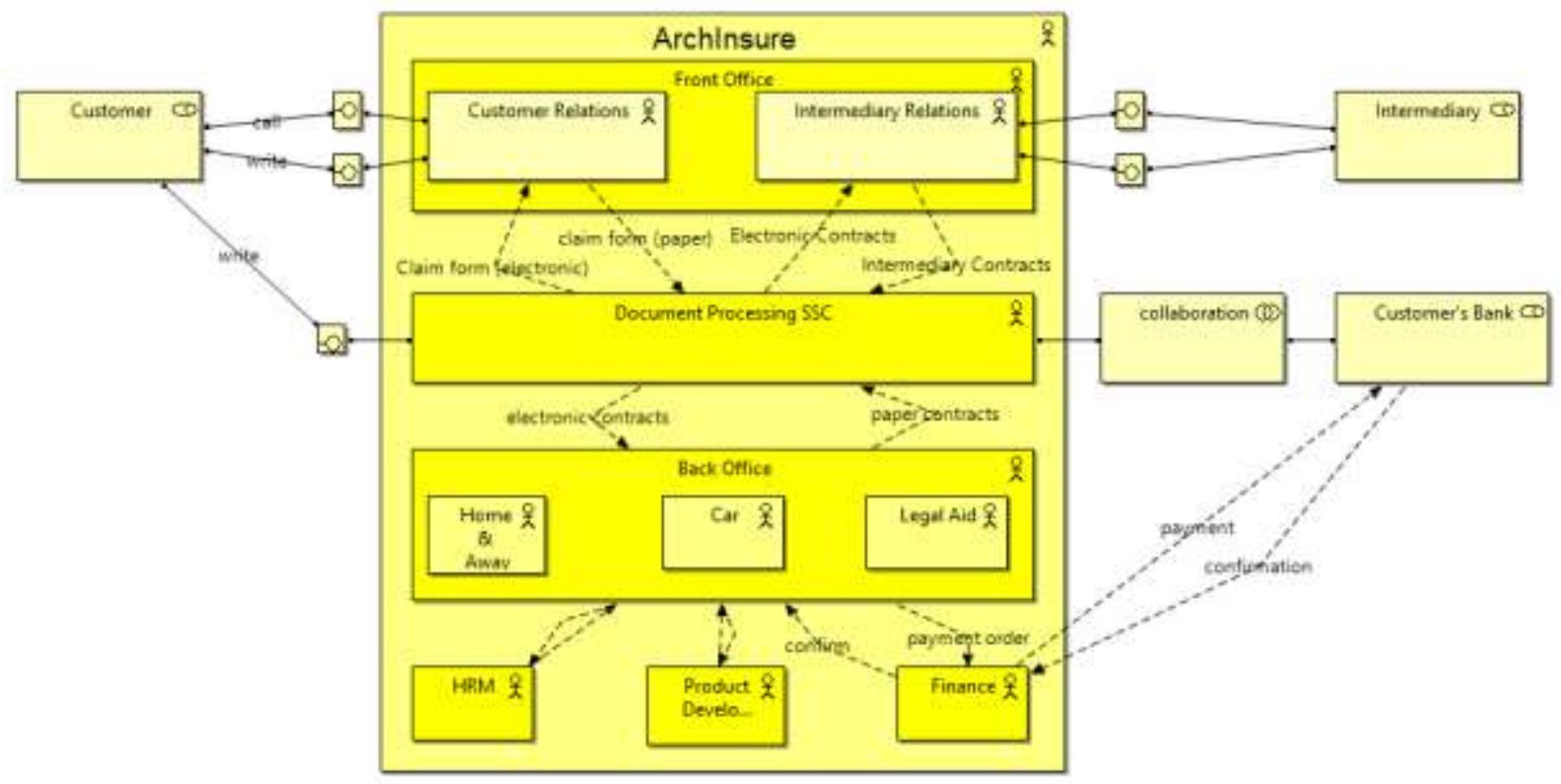

Figure 2: ArchInsure Actors Cooperation Perspective 
The ArchInsure Business Process Cooperation perspective is represented in Figure 2. The model depicts the interactive structure of the organizational unit including the front office, back office, service consumers and other intermediaries. Specifically, the nested ontology identifies roles, competencies and responsibilities of the organization units. The business process collaboration perspective is a high-level design of business processes that presents to the operational user deep perception into the flow of main business processes of the enterprise and their dependencies.

\subsection{ArchInsure Business Corporation Perspective}

The ArchiMate modelling language categorises business process and behavior based on an ordering of activities to realize categorization of a defined set of products or services. In ArchInsure ontology design, this is depicted in Figure 3.

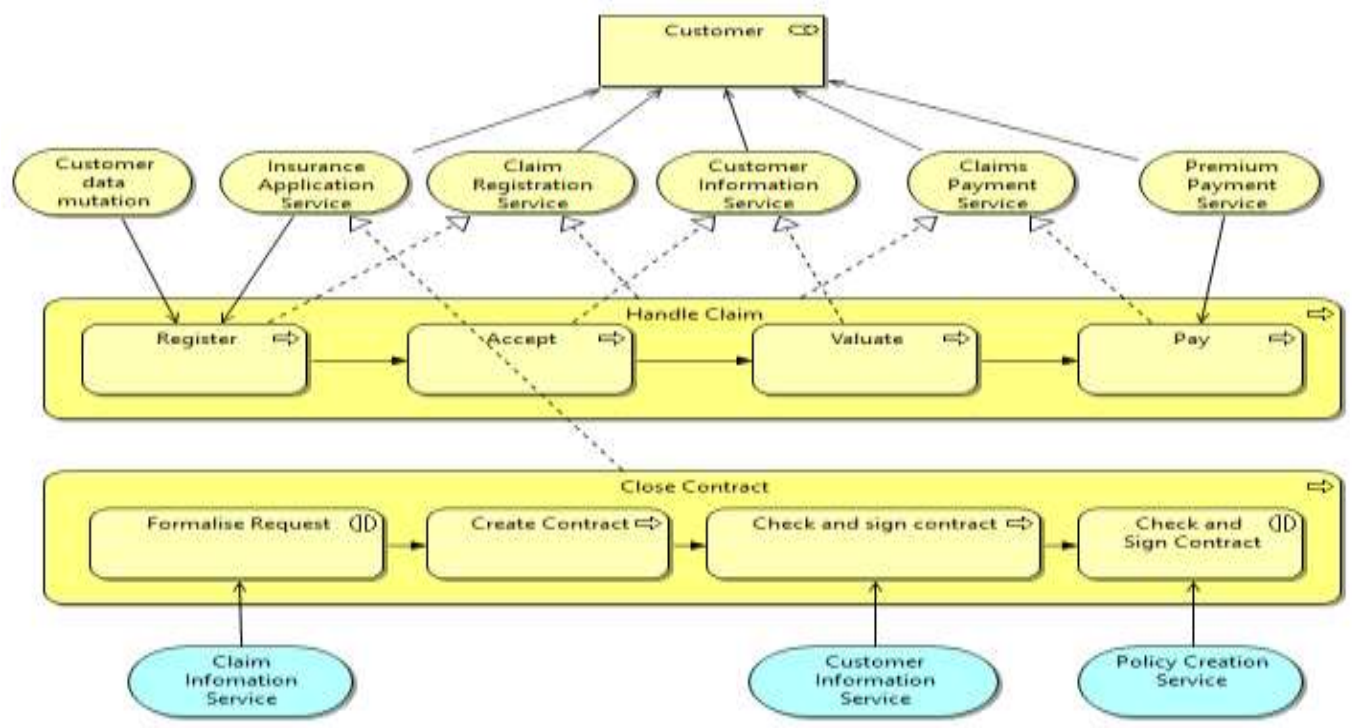

Figure 3: ArchInsure Business Corporation Perspective

The model decomposes the two fundamental business processes of ArchInsure into their high-level sub-processes: Handle Claim and Close Contract. Handle claim is executed when a claim for damage has been initiated by the service consumer. While the details of these processes may differ for divergent insurance product, the fundamental steps similar. The process architecture shows the most imperative business processes and their relationships, and distinctive phases within each of the processes at a higher level of abstraction.

\subsection{ArchInsure Business Function Perspectives}

The ArchiMate business function groups behavior based on a predetermined set of constraints. Business functions define the activities carried out by the enterprise and can be divided into primary functions and ancillary functions. The primary business functions that ArchInsure distinguishes are shown in Figure 4. The primary business functions are contained in the Business Role titled "Insurer" and categorize activities of the enterprise that yielding income. Ancillary business functions are support activities carried out by the enterprise in order to authenticate and facilitate the primary business functions activities.

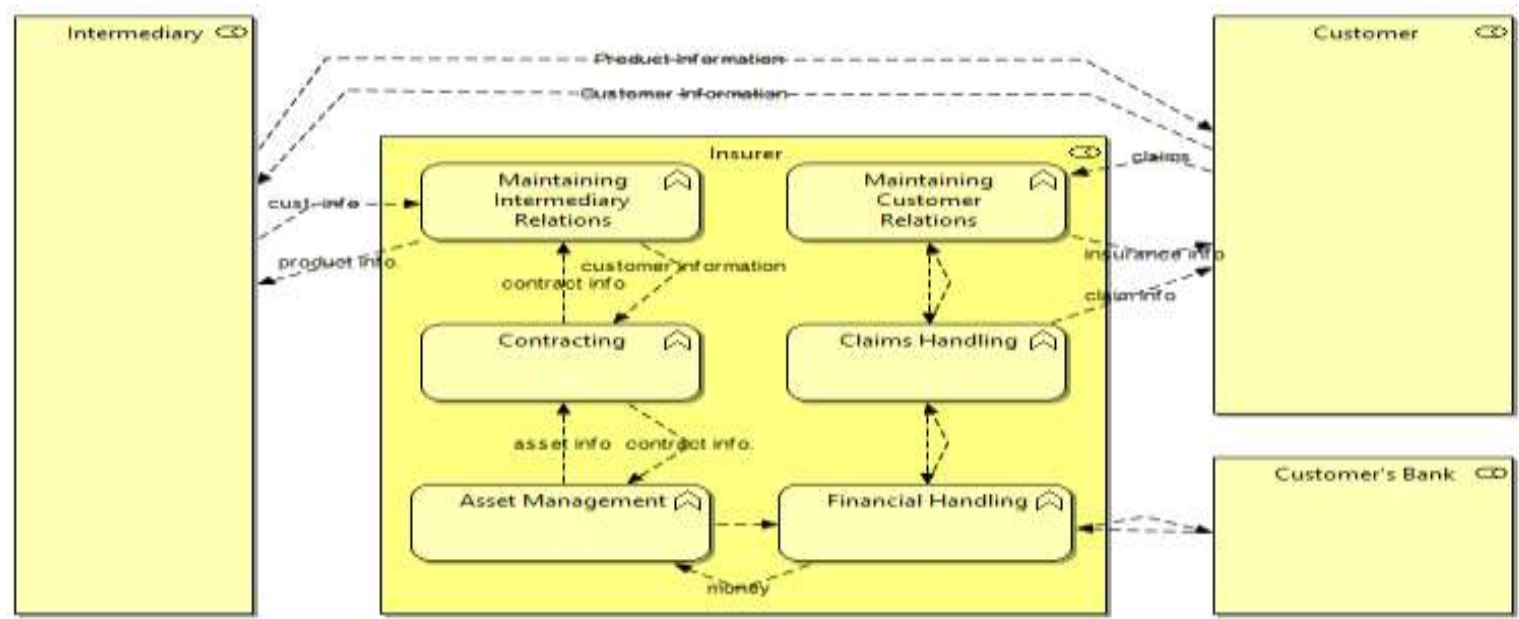

Figure 4: ArchInsure Business Function Perspective

These are represented as the business roles Intermediary, Customer and Customer's bank. As the main function of any insurance institution is to eliminate the uncertainty of an unexpected and sudden financial loss, the business function model 
depicted in Figure 4 is further synthesized into categories within business roles and enables the assessment of risk and financial administration of premiums for effective insurance coverage. The Business Function depicts a grouping of behavior based on resources.

\subsection{ArchInsure Business Process Perspectives}

The ArchInsure business process perspective depicts a reference model for business processes of an insurance institution comparable to a large repository of requirements for the industry. The processes interrelate with other business constructs, and specifically to the business component services that describe how technology can bolster requirements expressed in those processes. With the ArchInsure business process perspective, an insurer can contrast its own business processes to best practice business processes, to understand how to better support and categorize them for effective decision support systems. The business process modeled in Figure 5 shows a collection of linked tasks which find their end in the delivery of a service and product to a client. It defines a set of activities and tasks that, once completed, will enable the organizational goals to be actualized.

The process is annotated with distinctly annotated inputs and a singular output with the inputs made up of all of the dynamics that contribute either implicitly or explicitly to the added value of a service. The complex business process of ArchInsure can also be decomposed into several sub-processes, which have their own attributes as well as collaborative attributes that contribute towards actualizing the goal of the main-process. The outcome of a well-designed business process increases efficiency in terms of value for the consumer and increased proficiency which implies use of less resource.

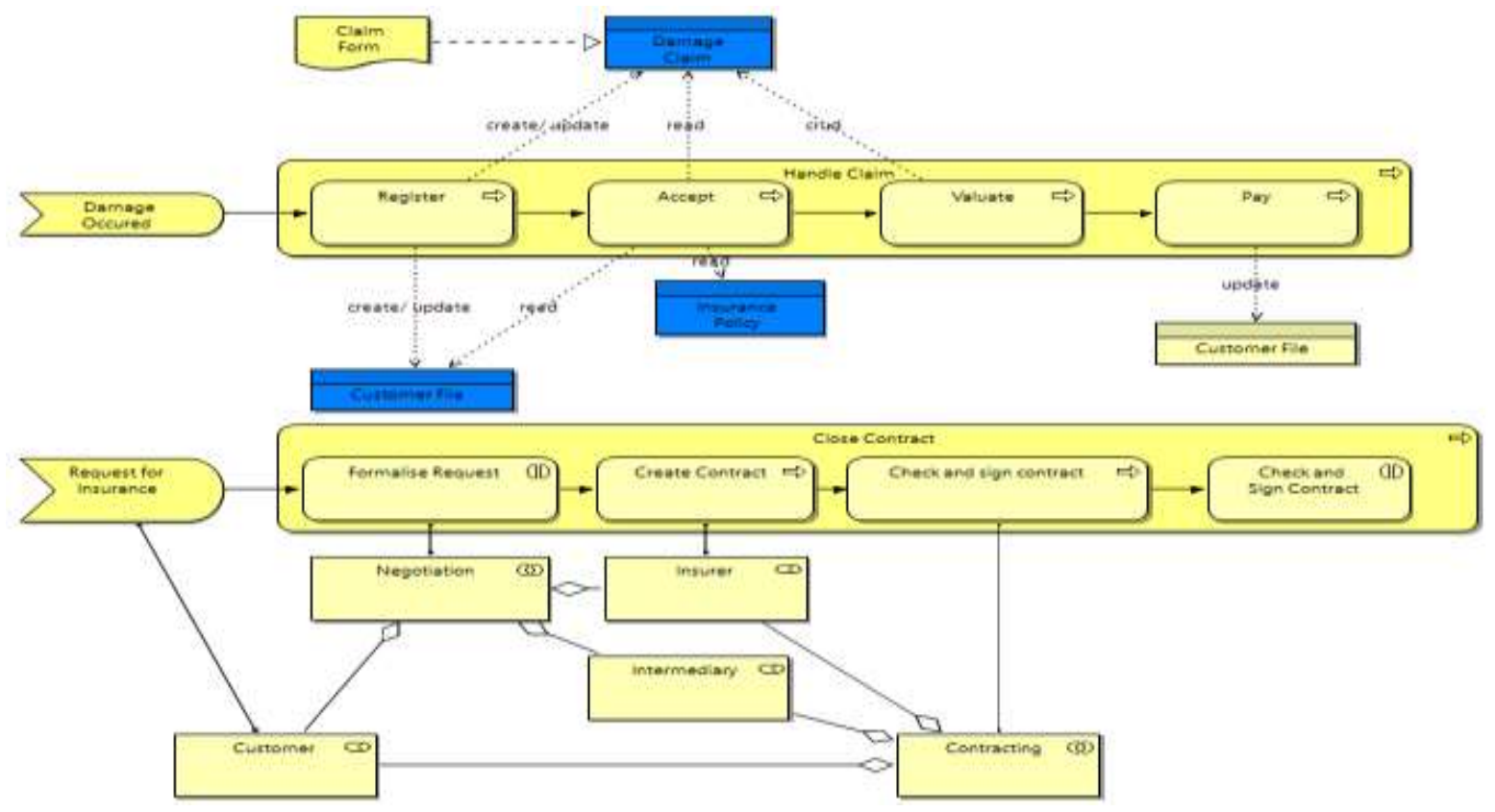

Figure 5: ArchInsure Business Process Perspective

In this ontology implementation, business function is a major organizational unit of a business and has specific responsibilities of execution of activities on a regular basis to enable the business actualize its goals. Function denotes both the organizational unit and the categories of activities performed. In contrast, business process postulates a set of activities, which draw on one or more business functions to help execute the organization's mission. Business Process as modeled in ArchInsure annotate artefacts directed at producing goals (service, product) while Business Function is adduced as a grouping of behavior based on resources. A direct composition of Business Functions and Business Processes as composite parts of each other would make this dissimilarity less meaningful [24]. Hence it can be inferred that the Business Process uses the Business Functions in some way instead of both being aggregates of each other.

\subsection{ArchInsure Information Structure Perspectives}

The ArchInsure Information Structure Perspective describes the major relationships between its theoretical business objects and its logical data artefacts. The purpose of the harmonization of the data objects in the Information Structure Perspective 
diagram shown in Figure 6 is to show the relationship between data entity, business service, and application components. The diagram shows how the logical entities are to be physically realized by application components. This allows effective scaling to be achieved and knowledge base of the system to be synchronized.

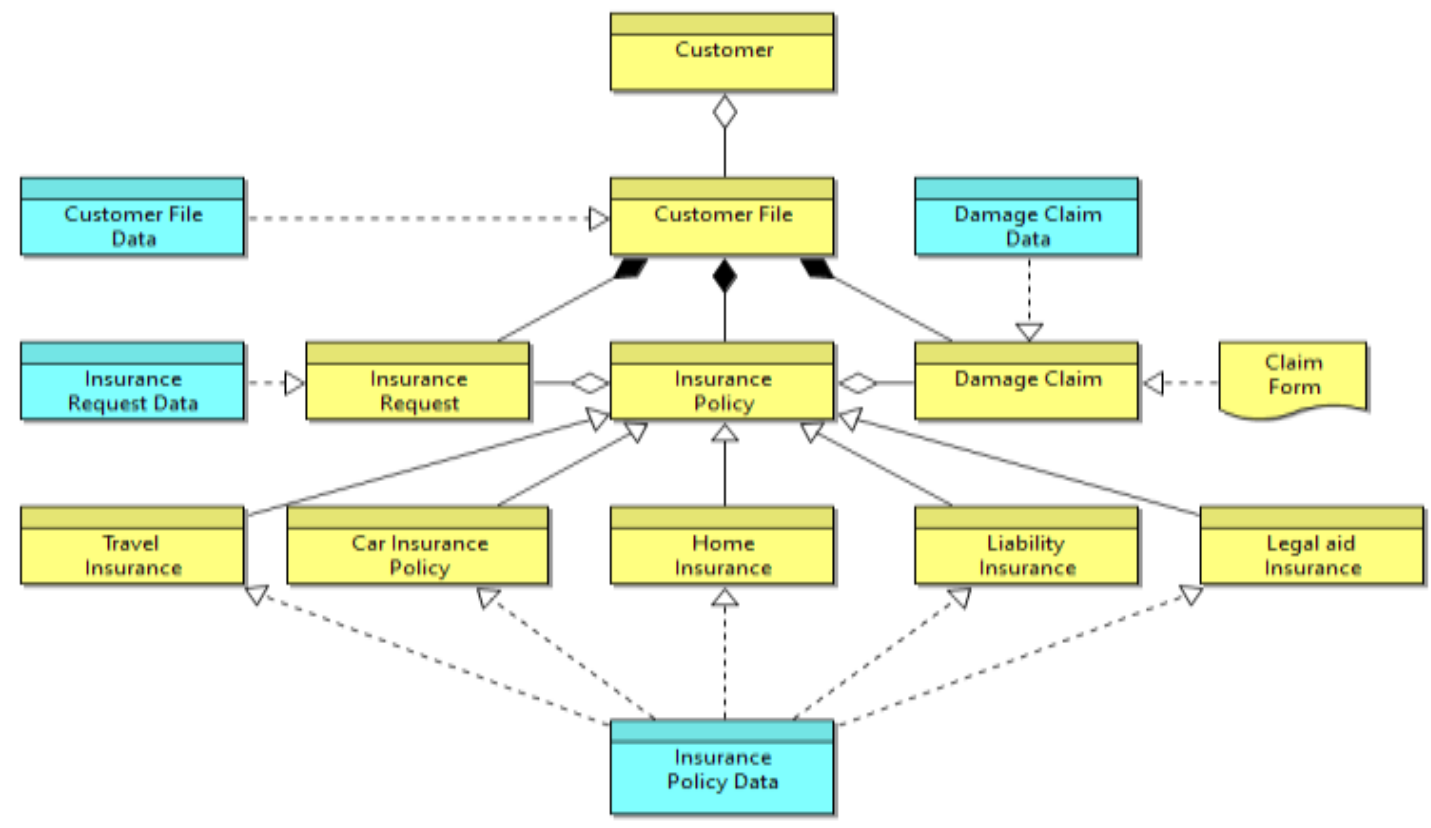

Figure 6: ArchInsure Information Structure Perspective

Also, by assigning business value to data, the complexity of the system can be simplified and the indication of the business criticality of application components can be gained.

\subsection{ArchInsure Service Realization Perspectives}

It is common for insurers to have heterogeneous modules that duplicate services and functionality across different systems implementations. The deleterious impact on maintenance and extensibility is very conspicuous when functionality needs to scaled. This emphasizes the need for the service realization perspective without which multiple systems will be impacted resulting in substantial duplication of effort. The ideal situation is to have a componentized solution where functionality is identified unambiguously and allocated to only one, component. This is annotated in the model in Figure 7. To evolve from an incongruent system with myriad of duplicated functionalities to a component-based knowledge categorization solution is by no means easy and requires a well-structured series of related ontologies.

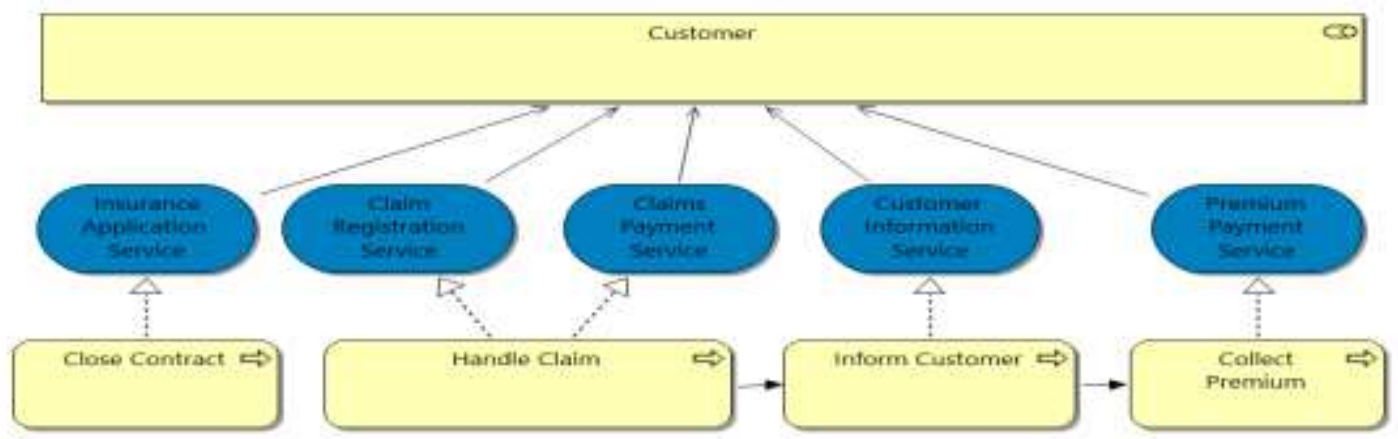

Figure 7: ArchInsure Service Realization Perspective

ArchInsure addresses this complex issue at the analytical and model design levels. This supports the primary business goals for knowledge-based system with improved flexibility of the systems and decreased disparity between the required business functionality thus facilitating decision support. 


\section{SUMMARY OF CONCLUSION}

In this paper, a framework that can be applied to deal with complex problems in insurance systems is presented. By traversing key findings across implementations, especially from the domain of ontologies, insurance and enterprise architectures with decision support frameworks, a step has been taken towards providing a model reference framework for the design and implementation of insurance system. Although many researchers in the fields of EA have investigated ways in which complex problem can be tackled in insurance systems, this work complements these efforts and contributes extensively by providing pragmatic ontologies for the implementation of such systems. This research fosters these efforts by providing a shared understanding of key domains and inputs for synergizing knowledge for solving complex problems in insurance companies and system developers working with a range of tools to solve challenges in the sector. The approach has initiated stimuli to calls for further research to address the complex challenge of evaluating the efficacy of complex problems and possibly assessing same through other numerous software validation and verification technics. With increased international public and private funding of insurance information systems and increased reliance on such applications, it is imperative to have an extensive and dependable approach that can drive their design. This paper has presented such mechanism to drive the relationship between classification and knowledge explored for complex problems of insurance categorization.

\section{REFERENCES}

[1] Rittel, Horst WJ, and Melvin M. Webber. "Dilemmas in a general theory of planning." Policy sciences 4.2 (1973): 155169.

[2] Hulme, Mike. Why we disagree about climate change: Understanding controversy, inaction and opportunity. Cambridge University Press, 2009.

[3] Australian Public Service Commission. "Tackling wicked problems: A public policy perspective." (2012).

[4] Levesque, Hector J. "Knowledge representation and reasoning." Annual review of computer science 1.1 (1986): 255287.

[5] Gibson, Cristina B. "From knowledge accumulation to accommodation: Cycles of collective cognition in work groups." Journal of Organizational Behavior: The International Journal of Industrial, Occupational and Organizational Psychology and Behavior 22.2 (2001): 121-134.

[6] Davies, Roy. "The creation of new knowledge by information retrieval and classification." Journal of documentation 45.4 (1989): 273-301.

[7] Ackrill, J. L. "Aristotle, Categories and De interpretation. Clarendon." (1963).

[8] DeGrace, Peter; Stahl, L. Hulet (1990). Wicked Problems, Righteous Solutions: A Catalog of Modern Engineering Paradigms. Yourdon Press. ISBN 978-0-13-590126-7.

[9] Conklin, Jeff (2003b). Dialog Mapping: An Approach for Wicked Problems. CogNexus Institute.

[10] Sølvberg, Arne; Kung, David (1993). An Introduction to Information Systems Engineering. Springer.

[11] Roberts, Nancy. "Wicked problems and network approaches to resolution." International public management review 1.1 (2000): 1-19.

[12] Shum, Simon Buckingham. "The roots of computer supported argument visualization." Visualizing argumentation. Springer, London, 2003. 3-24.

[13] Conklin, Jeff. "Dialog mapping: Reflections on an industrial strength case study." Visualizing argumentation. Springer, London, 2003. 117-136.

[14] Knapp, R. "Wholesome Design for Wicked Problems." The Public Sphere Project. http://www. publicsphereproject. org/content/wholesome-design-wicked-problems. Zugegriffen 15 (2008).

[15] Roemer, Milton Irwin. "Hospital utilization under insurance." Journal of Occupational and Environmental Medicine 2.5 (1960): 252.

[16] Frenk, Julio, and Avedis Donabedian. "State intervention in medical care: types, trends and variables." Health Policy and Planning 2.1 (1987): 17-31.

[17] Lankhorst, Marc M., Henderik Alex Proper, and Henk Jonkers. "The architecture of the archimate language." Enterprise, business-process and information systems modeling. Springer, Berlin, Heidelberg, 2009. 367-380.

[18] Wendt, Claus, Lorraine Frisina, and Heinz Rothgang. "Healthcare system types: a conceptual framework for comparison." Social Policy \& Administration 43.1 (2009): 70-90.

[19] Roemer, Milton Irwin. "Hospital utilization under insurance." Journal of Occupational and Environmental Medicine 2.5 (1960): 252.

[20] Terris, Milton. "The three world systems of medical care: trends and prospects." American Journal of Public Health 68.11 (1978): 1125-1131. 
International Journal of Advances in Scientific Research and Engineering (ijasre), Vol 6 (5), May-2020

[21] Wendt, Claus, Lorraine Frisina, and Heinz Rothgang. "Healthcare system types: a conceptual framework for comparison." Social Policy \& Administration 43.1 (2009): 70-90.

[22] Frenk, Julio, and Avedis Donabedian. "State intervention in medical care: types, trends and variables." Health Policy and Planning 2.1 (1987): 17-31.

[23] Olakunde, Babayemi O. "Public health care financing in Nigeria: which way forward?." Annals of Nigerian Medicine 6.1 (2012): 4.

[24] Josey, Andrew, et al. "An introduction to the ArchiMate ${ }^{\circledR} 3.0$ specification." White Paper from The Open Group (2016). 